\section{Development of subsquamous cancer after hybrid endoscopic therapy for intramucosal Barrett's cancer}

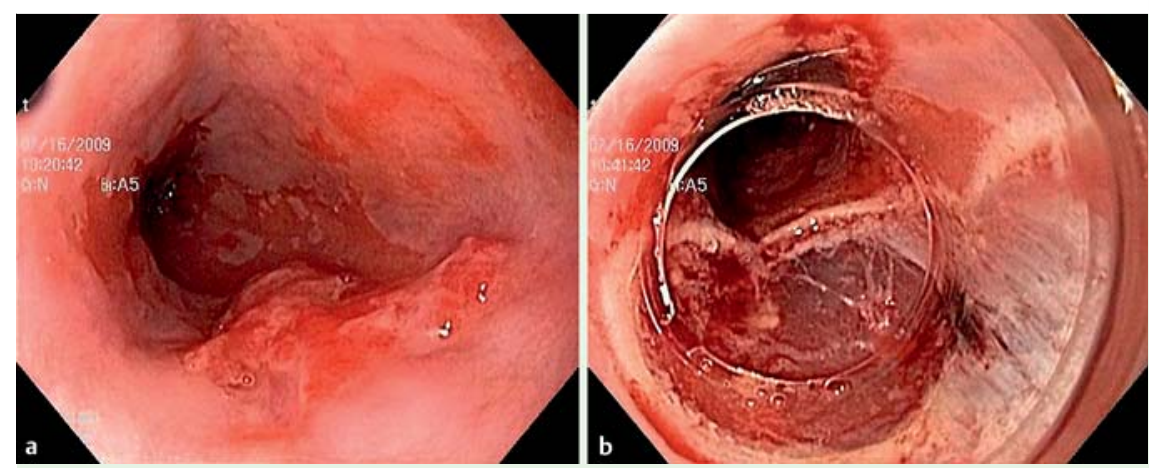

Fig. 1 a On initial endoscopy at our institution, a long-segment Barrett's esophagus was seen from $40 \mathrm{~cm}$ to islands at $35 \mathrm{~cm}$. A visible lesion extended from 37 to $35 \mathrm{~cm}$ from the incisors. b Saline-assisted mucosal resection was performed. Pathology confirmed the presence of intramucosal carcinoma and high grade dysplasia in the setting of Barrett's esophagus.
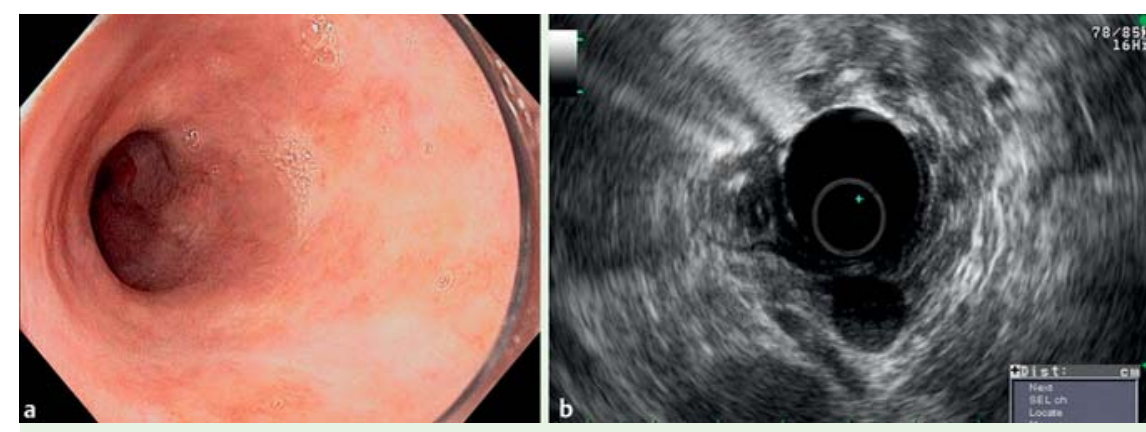

Fig.2 a Follow-up endoscopy post hybrid therapy demonstrated neosquamous epithelium without other visible lesions. $\mathbf{b}$ On endoscopic ultrasound, one abnormal lymph node was visualized in the gastrohepatic ligament. Fine needle aspiration of the lymph node was positive for adenocarcinoma.
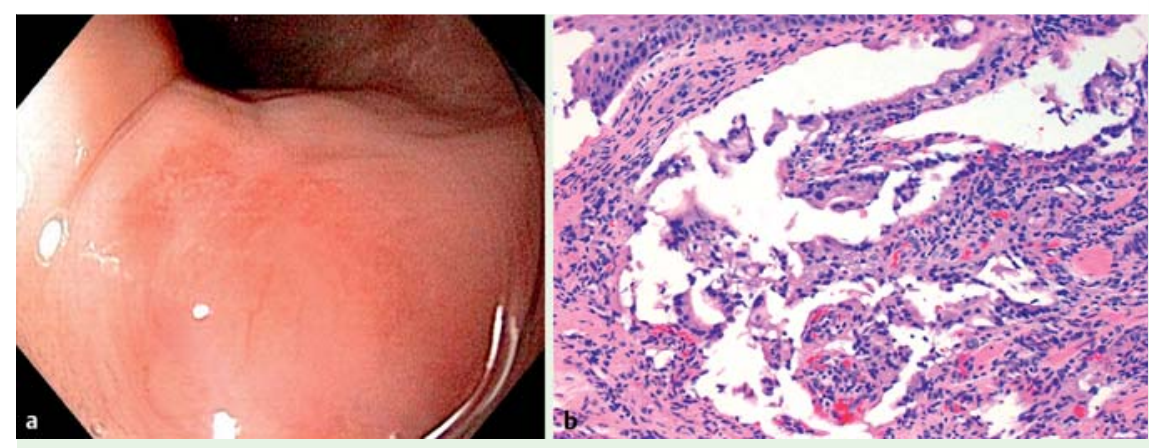

Fig. 3 Procedure number 6 (32 months). a A localized discolored mucosa was found in the esophagus with otherwise unremarkable neosquamous epithelium. Biopsies were taken. $\mathbf{b}$ Sections of the biopsy from discolored mucosa revealed dysplastic glandular epithelium buried beneath intact normal squamous mucosa. The dysplastic glands exhibited a complex architecture and high grade cytologic features, including nuclear hyperchromasia, high nuclear to cytoplasm (N: C) ratio, and loss of normal nuclear polarity. The features are consistent with high grade dysplasia or intramucosal carcinoma.
A 60-year-old man presented for endoscopic management of a long-segment Barrett's esophagus with a visible lesion (๑ Fig. 1). Endoscopic mucosal resection (EMR) of the lesion demonstrated intramucosal carcinoma (IMC) without submucosal invasion on histological examination. Endoscopic ultrasound (EUS) revealed no malignant adenopathy. Three subsequent sessions were performed with focal EMR of all visible lesions and radiofrequency ablation (RFA) of the remnant Barrett's esophagus segment. On a follow-up endoscopy 2 years after the initial intervention, neosquamous epithelium with no apparent Barrett's esophagus was seen and a rigorous biopsy protocol did not reveal any occult cancer. However, EUS demonstrated a 10-mm abnormal-looking gastrohepatic lymph node, diagnosed as metastatic adenocarcinoma by fine needle aspiration ( $\bullet$ Fig. 2). Subsequent computed tomography (CT) scan and colonoscopy did not demonstrate a primary site. A repeat esophagogastroduodenoscopy/EUS performed 6 months later demonstrated squamous mucosa with focal discoloration in the mid esophagus. Biopsies revealed neoplasia buried under squamous epithelium ( $\bullet$ Fig.3).

This case demonstrates a subsquamous Barrett's adenocarcinoma after hybrid endoscopic therapy, leading to metastatic disease. The goals of endoscopic therapy of Barrett's-associated neoplasia are to completely eradicate the known neoplasia and also treat the remainder of the at-risk epithelium. A hybrid approach, where EMR is used to eradicate and stage all visible lesions, and RFA is used to treat the remnant Barrett's esophagus, is an appropriate management strategy $[1,2]$.

Subsquamous Barrett's esophagus has been noted both pre and post ablation in the literature $[3,4]$. Although the reported incidence of subsquamous Barrett's esophagus is relatively low, the true incidence and risk of progression to neoplasia might be underestimated. The size and depth of biopsy specimens may be insufficient to determine whether there are buried glands [5]. Furthermore, there is inherent sampling error from biopsy protocols. More studies on biopsy protocols and long-term outcomes after endotherapy are required to elucidate the risk of subsquamous Barrett's esophagus and neoplasia.

Endoscopy_UCTN_Code_CCL_1AB_2AC_3AB 
V. J. A. Konda, M. Gonzalez Haba Ruiz, J. Hart, I. Waxman

Center for Endoscopic Research and Therapeutics, Section of Gastroenterology, University of Chicago Medical Center, Chicago, Illinois, USA

\section{References}

1 Pouw RE, Wirths K, Eisendrath P et al. Efficacy of radiofrequency ablation combined with endoscopic resection for Barrett's esophagus with early neoplasia. Clin Gastroenterol Hepatol 2010; 8: 23-29

2 van Vilsteren $F G$, Pouw RE, Seewald $S$ et al. Stepwise radical endoscopic resection versus radiofrequency ablation for Barrett's oesophagus with high-grade dysplasia or early cancer: a multicentre randomised trial. Gut 2011; 60: 765-773

3 Chennat J, Ross AS, Konda VJ et al. Advanced pathology under squamous epithelium on initial EMR specimens in patients with Barrett's esophagus and high-grade dysplasia or intramucosal carcinoma: implications for surveillance and endotherapy management. Gastrointest Endosc 2009; 70: 417-421

4 Gray NA, Odze RD, Spechler SJ. Buried metaplasia after endoscopic ablation of barrett's esophagus: a systematic review. Am J Gastroenterol 2011; 106: 1899-1908

5 Gupta N, Mathur SC, Dumot JA et al. Adequacy of esophageal squamous mucosa specimens obtained during endoscopy: are standard biopsies sufficient for postablation surveillance in Barrett's esophagus? Gastrointest Endosc 2012; 75: 11 - 18
Bibliography

Dol http://dx.doi.org/

10.1055/s-0032-1310139

Endoscopy 2012; 44: E390-E391

(C) Georg Thieme Verlag KG

Stuttgart · New York

ISSN 0013-726X

\section{Corresponding author}

\section{Waxman, MD}

Center for Endoscopic Research and Therapeutics (CERT)

University of Chicago

5758 S. Maryland Avenue, MC 9028

Chicago

IL 60637

USA

Fax: +1-773-834-8891

iwaxman@medicine.bsd.uchicago.edu 